\section{Another piece in the 'research mosaic' that describes the role of the lung microbiome in COPD}

\author{
Gary B Huffnagle ${ }^{1,2}$
}

Bacterial colonisation/infection is ubiquitous in patients with COPD and is likely a biologically relevant facet of disease progression. Correlations have been reported between the identification of bacteria and increases in the intensity of the inflammatory/immune response, cough, sputum production and rates of acute exacerbations of COPD. ${ }^{1-5}$ The inflammatory/ immune response in COPD also persists despite smoking cessation and there is a particularly enhanced inflammatory response in chronic bronchitis and acute exacerbations of COPD, all of which is consistent with a microbial mechanism underlying chronicity and exacerbations. Chronic bacterial colonisation in COPD subjects had been traditionally evaluated by cultivation-based methods of sputum and bronchoalveolar lavage (BAL), with the magnitude and types of organisms varying widely. Culture-independent analyses, such as those used in the study by Einarsson et $a l,{ }^{6}$ are providing new insights into the pulmonary microbiome of patients with COPD. This current study underscores the concept that the COPD lung harbours a microbiome distinct from that in healthy non-smokers and smokers, which is not captured completely by standard culture techniques. Overall, this study, together with complementary studies from other labs, ${ }^{6-13}$ is building a 'research mosaic' that is identifying the changes in the microbiome that occur during COPD and delineating their importance in the pathogenesis of the disease.

In the paper by Einarsson et $a l,{ }^{6}$ the objective was to determine whether there are differences in the lower airway

\footnotetext{
${ }^{1}$ Division of Pulmonary and Critical Care Medicine, Department of Internal Medicine, University of Michigan Medical School, Ann Arbor, Michigan, USA; ${ }^{2}$ Department of Microbiology and Immunology, University of Michigan Medical School, Ann Arbor, Michigan, USA

Correspondence to Dr Gary B. Huffnagle, Division of Pulmonary and Critical Care Medicine, Department of Internal Medicine, University of Michigan Medical School, 6301 MSRB III-Box 5642, 1150 W. Medical Center Drive, Ann Arbor Ml 48109-5642, USA; ghuff@umich.edu
}

microbiota between subjects with COPD, smokers and non-smokers. Lower airway samples in the study were obtained by BAL and the microbiota determined by both culture-based and cultureindependent analyses, using Illumina MiSeq sequencing, targeting the 16S rRNA gene in bacteria. Both bacterial load and measurements of community composition were performed in this study. There is one secondary readout of this study that should be emphasised: culturebased analysis of the subjects clearly demonstrated that viable bacteria, both aerobic and anaerobic, were isolated from the lungs of non-smokers, as well as healthy smokers and subjects with COPD. This is consistent with other recent studies of the healthy lung microbiome, including those from our group, ${ }^{14-17}$ and the myth that the healthy lungs are 'free from bacteria' should be laid to rest once and for all, as we have discussed in more detail recently. ${ }^{18}$

The study by Einarsson et $a l^{6}$ also reported that for the majority of patients with COPD, the lung microbiota is significantly different than that present in individuals without disease, including healthy smokers. It has been documented that, surprisingly, smoking in and of itself does not alter the lung microbiome. ${ }^{17}$ The observation that the lung microbiota is different during COPD is consistent with a number of other studies in the field that have used a variety of sample types. ${ }^{7-13}$ However, what is emerging in the field is the concept that changes in overall bacterial community diversity during COPD do not necessarily follow a straight progression of either increasing diversity or decreasing diversity during the progression of disease. Community diversity may increase at some stages, perhaps owing to impaired clearance of microaspirated anaerobes from the upper airways. At other stages, such as during advanced disease, community diversity may decrease as a result of the emergence of a pathogen that numerically dominates the diseased COPD lung.

However, it is also important to re-emphasise the need for proper technical controls in any sequencing-based study of metagenomic DNA samples in which the target DNA is of low abundance, such as for lower airway metagenomic DNA. The study by Salter et al ${ }^{19}$ clearly demonstrated how 'diversity' in a sample will increase as the biomass drops because the ratio of background reagent signal to target signal increases. The reagents for DNA isolation, amplification and the sequencing reaction will all generate sequence reads at low target DNA concentrations that create problems in separating true biological signal from noise. The study by Einarsson et $a l^{6}$ provided information in the online supplement to address the background issue. It is my opinion that all the respiratory journals should require robust technical controls for culture-independent microbiome analysis, especially when significant conclusions are based on either (1) the statistically significant occurrence of a rare organism or (2) changes in community diversity that are attributed to changes in rare operational taxonomic units (ie, the length of the community membership 'tail' changes). These data can be included in online supplements but it is important for the validity of the study.

This study used BAL samples, which are robust samples for the lower airways. To date, our laboratory has predominantly used BAL samples for our studies of the lung microbiome. ${ }^{14} 15{ }^{20}$ However, there is no single analysis of the airway microbiome that provides a complete picture of the microbiology of the lungs. The lungs are difficult to study longitudinally and cross-sectionally. Total BAL, sputum, cellfree BAL supernatants and biopsies are all samples that provide different insights into the processes of lung microbiology. In addition, different types of culturebased and molecular analyses add to the larger story that includes changes in microbial composition, total colonisation levels, macro-anatomic and microanatomic heterogeneity, culturability and changes in microbial gene expression and metabolism, among other things. The ultimate outcome under study is a change in the health status of the host. The combination of both culture and molecular analyses is a key feature of this current study and one that should be increasingly used in future microbiome analyses of respiratory health and disease.

One other emerging concept supported by this study is that there is no 'smoking gun' for a bacterial species that is associated with worsening disease in COPD. It was observed that there is a loss of the bacteria 
typically found in healthy individuals such as Prevotella and Veillonella ${ }^{14} 17$ and scattered acquisition of more well-known COPD-associated pathogens such as those within the Pseudomonas, Haemophilus and Streptococcus species. The paper contains data that are consistent with a model of a change in the lung environment during COPD that favours the outgrowth of a microbe and perpetuation of the inflammatory processes, rather than a transmissible infectious process.

One of the admitted limitations of this study is the sample size. In fact, relatively small sample sizes have plagued all BAL microbiome studies in COPD. With the cost of sequencing having dropped and the methodologies (procedural and bioinformatic) having been vetted, studies such as those by Einarsson et $a l^{6}$ and others are providing the rationales for much larger cohort analyses of the relationship between changes in the lower airway (BAL) lung bacterial microbiome and clinical parameters in COPD. These are the studies that will be needed to glue down this developing research mosaic that depicts the role of the lung microbiome in COPD pathogenesis.

Competing interests None declared.

Provenance and peer review Commissioned; externally peer reviewed.

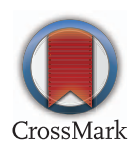

To cite Huffnagle GB. Thorax 2016;71:777-778.

Published Online First 19 July 2016

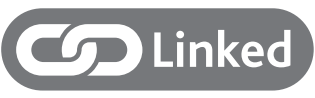

http://dx.doi.org/10.1136/thoraxjnl-2015-207235

Thorax 2016;71:777-778.

doi:10.1136/thoraxjnl-2015-207415

\section{REFERENCES}

1 Sethi S, Maloney J, Grove L, et al. Airway inflammation and bronchial bacterial colonization in chronic obstructive pulmonary disease. Am J Respir Crit Care Med 2006;173:991-8.

2 Hill AT, Campbell EJ, Hill SL, et al. Association between airway bacterial load and markers of airway inflammation in patients with stable chronic bronchitis. Am J Med 2000;109:288-95.

3 Martinez FJ, Han MK, Flaherty K, et al. Role of infection and antimicrobial therapy in acute exacerbations of chronic obstructive pulmonary disease. Expert Rev Anti Infect Ther 2006;4:101-24.

4 Sethi S, Murphy TF. Infection in the pathogenesis and course of chronic obstructive pulmonary disease. N Engl J Med 2008;359:2355-65.

5 Taylor D, Clancy R, Cripps A, et al. An alteration in the host-parasite relationship in subjects with chronic bronchitis prone to recurrent episodes of acute bronchitis. Imm Cell Biol 1994;72:143-51.

6 Einarsson GG, Comer DM, Mcllreavey L, et al. Community dynamics and the lower airway microbiota in stable chronic obstructive pulmonary disease, smokers and healthy non-smokers. Thorax 2016;71: 795-803.

7 Huang YJ, Kim E, Cox MJ, et al. A persistent and diverse airway microbiota present during chronic obstructive pulmonary disease exacerbations. OMICS 2010;14:9-59.

8 Huang YJ, Sethi S, Murphy T, et al. Airway microbiome dynamics in exacerbations of chronic obstructive pulmonary disease. I Clin Microbiol 2014;52:2813-23.

9 Molyneaux PL, Mallia P, Cox MJ, et al. Outgrowth of the bacterial airway microbiome after rhinovirus exacerbation of chronic obstructive pulmonary disease. Am J Respir Crit Care Med 2013;188:1224-31.

10 Cabrera-Rubio R, Garcia-Núñez M, Setó L, et al. Microbiome diversity in the bronchial tracts of patients with chronic obstructive pulmonary disease. J Clin Microbiol 2012;50:3562-8.

11 Garcia-Nuñez M, Millares L, Pomares X, et al. Severity-related changes of bronchial microbiome in chronic obstructive pulmonary disease. I Clin Microbiol 2014;52:4217-23.

12 Pragman AA, Kim HB, Reilly CS, et al. The lung microbiome in moderate and severe chronic obstructive pulmonary disease. PLoS One 2012;7: e47305.

13 Erb-Downward JR, Thompson DL, Han MK, et al. Analysis of the lung microbiome in the "healthy" smoker and in COPD. PLoS One 2011;6: e16384.

14 Bassis CM, Erb-Downward JR, Dickson RP, et al. Analysis of the upper respiratory tract microbiotas as the source of the lung and gastric microbiotas in healthy individuals. mBio 2015;6:e00037.

15 Dickson RP, Erb-Downward JR, Freeman CM, et al. Changes in the lung microbiome following lung transplantation include the emergence of two distinct pseudomonas species with distinct clinical associations. PLOS ONE 2014;9:e97214.

16 Segal LN, Alekseyenko AV, Clemente JC, et al. Enrichment of lung microbiome with supraglottic taxa is associated with increased pulmonary inflammation. Microbiome 2013;1:19.

17 Morris A, Beck JM, Schloss PD, et al. Comparison of the respiratory microbiome in healthy nonsmokers and smokers. Am J Respir Crit Care Med 2013;187:1067-75.

18 Dickson RP, Erb-Downward JR, Martinez FJ, et al. The microbiome and the respiratory tract. Annu Rev Physiol 2016;78:481-504.

19 Salter SJ, Cox MJ, Turek EM, et al. Reagent and laboratory contamination can critically impact sequence-based microbiome analyses. BMC Biol 2014;12:87.

20 Dickson RP, Erb-Downward JR, Freeman CM, et al. Spatial variation in the healthy human lung microbiome and the adapted island model of lung biogeography. Ann Am Thorac Soc 2015;12:821-30. 